\title{
Transient extensional rheology of wormlike micelle solutions
}

\author{
Jonathan P. Rothstein ${ }^{\text {a) }}$ \\ Department of Mechanical and Industrial Engineering, University \\ of Massachusetts, Amherst, Massachusetts 01003
}

(Received 27 February 2003; final revision received 1 July 2003)

\begin{abstract}
Synopsis
A filament stretching rheometer is used to follow the evolution in the tensile force and the flow induced birefringence of a wormlike micelle solution experiencing a uniaxial elongation flow. The experiments are performed using a series of wormlike micelle solutions of cetyltrimethylammonium bromide and sodium salicylate in de-ionized water. The linear viscoelastic shear rheology of the wormlike micelle solutions is well described by an upper convected Maxwell model with a single relaxation time. In transient homogeneous uniaxial extension, the wormlike micelle solutions demonstrate significant strain hardening and a failure of the stress-optical, however, no stress-conformation hysteresis is observed. A quantitative fit to the extensional rheology of each of the wormlike micelle solution tested is achieved with a FENE-PM model having as few as two relaxation modes. At a critical stress, nearly independent of strain rate, the wormlike micelle solutions filaments are found to fail through a dramatic rupture near the axial midplane. This filament failure is not the result of elastocapillary thinning as is commonly observed in the filament stretching of weakly strain hardening polymer solutions. Instead, the filament failure might stem from the local scission of individual wormlike micelle chains. The energy of wormlike micelle chain scission is calculated to be roughly $4 k_{B} T$ for all the solutions tested, nearly independent of both the imposed extension rate and the concentration of the surfactant and the salt. (C) 2003 The Society of Rheology. [DOI: 10.1122/1.1603242]
\end{abstract}

\section{INTRODUCTION}

Surfactants are molecules that consist of a hydrophilic head group and a hydrophobic tail. When dissolved in water they can spontaneously self-assemble to form several different types of aggregates [Israelachvili (1985); Larson (1999); Rehage and Hoffmann (1991)]. The morphologies of these aggregates can range in shape and size from spherical micelles, to wormlike micelles, to lamellae depending on surfactant and counterion concentration. Because of their extensive use in everything from additives to paints and detergents to oil recovery and pesticides, a considerable amount of research has been devoted to investigating the morphology, phase transitions, and shear rheology of different surfactant solutions [Cates (1987); Olmsted (1999); Rehage and Hoffmann (1991); Shikata and Kotaka (1991); van Egmond (1998)]. Under the proper conditions, the micelles, resembling slender rods, can entangle and impart viscoelasticity to the fluid [Cates (1987)]. The behavior of wormlike micelles solutions is similar to that of polymer solutions and melts. The primary difference being that unlike a covalently bonded polymer

a)Electronic mail: rothstein@ecs.umass.edu

(C) 2003 by The Society of Rheology, Inc.

J. Rheol. 47(5), 1227-1247 September/October (2003) 0148-6055/2003/47(5)/1227/21/\$25.00

1227 
backbone, micelles are in a state of thermodynamic equilibrium with the solvent and are perpetually broken and reformed under Brownian fluctuations. This leads to a broad and dynamic distribution of micelle lengths which, as proposed by Cates and Turner (1990), can change under an imposed shear or extensional flow.

Studies have shown that in the regime of linear deformation imposed by small amplitude oscillatory shear flows, wormlike micelle solutions can be accurately modeled by a Maxwell model having just one or two relaxation times. Cates proposed that, in the fast breaking-limit, the relaxation time, $\lambda=\left(\lambda_{\text {break }} \lambda_{d}\right)^{1 / 2}$, is a function of the reptation or disentanglement time of the micelle in solution, $\lambda_{d}$, and the average lifetime of the micelle, $\lambda_{\text {break }}$ [Cates (1987); Rehage and Hoffmann (1991)]. The dynamical response of polymeric fluids and micelle solutions in extension is quite different than in simple shear and it is unclear whether one should expect a similar single mode response.

The steady shear rheology of wormlike micelle solutions is similar to the rheology of viscoelastic polymer solutions and melts under most conditions [Rehage and Hoffmann (1991)]. However, the imposition of very large stresses and strains on wormlike micelle solutions can result in a wealth of complex phenomena. In some viscometric flows, wormlike micelle solutions have been found to exhibit a dramatic shear thickening behavior often followed by the onset of a flow instability [Hu et al. (1998); Kadoma and van Egmond (1998); Liu and Pine (1996); van Egmond (1998); Wheeler et al. (1998)]. While in other shear flows, the formation of banded structures or "slip layers" of different surfactant morphologies having dramatically different rheological properties has been observed [Hu et al. (1998); Larson (1992); Mair and Calaghan (1996)]. These phenomena have been shown to originate from shear-induced structure formation, shear-induced phase change from an isotropic to a nematic state, shear-induced gelation and shearinduced demixing [Berret et al. (1998); Kadoma and van Egmond (1998); Liu and Pine (1996); Wheeler et al. (1998)]. In flows of wormlike micelle solutions with strong extensional components, such as the flow past a falling sphere or a rising bubble, new flow instabilities have recently been observed that appear to originate from within the regions of the strong extensional flow in the wake of the sphere and bubble [Belmonte (2000); Chen and Rothstein (2002)]. To understand and eventually predict the behavior of wormlike micelle solutions in flows with strong extensional components, such as the flow past a sedimenting sphere or the flow through porous media, it is necessary to first understand the behavior of wormlike micelle solutions in homogenous uniaxial extensional flows.

Extensional rheology has recently become a topic of great interest and importance to the complex fluids community. The extensional behavior of polymeric fluids, especially dilute polymer solutions, has received a considerable amount of attention in recent years [McKinley and Sridhar (2002)], however, little is known about the behavior of wormlike micelles solutions in extensional flows, in particular transient flows. Devices like the four roll mill and the opposed jet device have been used in the past to investigate the extensional behavior of wormlike micelle solutions [Chen and Warr (1997); Fischer et al. (1997); Lu et al. (1998); Prud'homme and Warr (1994); Walker et al. (1996)]. Unfortunately, these devices are plagued by an unknown prestrain history, some degree of shearing in the flow field and the inability to make transient extensional rheology measurements. The filament stretching rheometer has emerged as an accurate device for reproducibly measuring the response of a fluid to an imposed transient homogeneous uniaxial extensional flow field [Anna et al. (2001)] and will be used exclusively in the experiments presented in this manuscript.

Prud'homme and Warr (1994) were the first to perform a comprehensive study of the extensional rheology of wormlike micelle solutions. They studied a series of equimolar tetradecyltrimethylammonium bromide (TTABr) sodium salicylate ( $\mathrm{NaSal}$ ) solutions in 
both the dilute and semidilute regime using a Rheometrics RFX opposed jet device. The response of the wormlike micelle solutions can be understood best within the framework of polymer reptation theory [Larson (1999)]. At low Deborah numbers, below the coilstretch transition, a plateau in the steady-state extensional viscosity was observed corresponding to the Newtonian response. Unlike entangle polymer melts [Bhattacharjee et al. (2002)], for which tube orientation has been found to result in extension-rate thinning, no such decrease in the steady state extensional viscosity was observed as the Deborah number was increased. At higher extension rates, chain stretching within the oriented segments was observed to lead to strain-hardening in the extensional rheology. At a critical Deborah number, the extensional viscosity was observed to reach a maximum and decreases with further increases in Deborah number. Unfortunately, the data above this critical Deborah number are marred by the onset of a flow instability and the ejection of strongly birefringent fluid from the stagnation region [Prud'homme and Warr (1994)]. Even with the problems in the stagnation flow field, Prud'homme and Warr (1994) theorized that the observed reduction in the extensional viscosity at high extension rates was the result of a scission of the wormlike micelles in the strong extensional flow. The hypothesis of Prud'homme and Warr was later supported by light scattering measurements [Chen and Warr (1997)] which clearly demonstrated a decrease in micelle radius of gyration coinciding with the onset of extensional viscosity thinning.

In the experiments presented in this manuscript, a filament stretching rheometer was used to follow the evolution in the tensile force and the flow induced birefringence of a series of wormlike micelle solutions experiencing an uniaxial elongation flow. The experiments were performed using a series of wormlike micelle solutions of cetyltrimethylammonium bromide (CTAB) and NaSal in de-ionized water. To our knowledge no transient extensional rheology measurements have been made on wormlike micelle solutions to date. These measurements will help us obtain a better understanding of the timedependent response of these fluids to strong flows and develop adequate constitutive models for possible future use in numerical simulations. Additionally, this research will allow us to determine whether the extensional viscosity results obtained with an opposed jet device, especially the high Deborah number results, can be reproduced within a filament stretching rheometer or whether they are an artifact of the nonhomogeneous flow created within the opposed jet device.

The outline of this paper is as follows. In Sec. II, we briefly describe the implementation of the filament stretching rheometer, the flow induced birefringence optical train and the test fluids used. In Sec. III A, we discuss the shear rheology of the test fluids. In Sec. III B we discuss the transient homogeneous uniaxial extensional rheology of the test fluids. Finally, in Sec. IV we conclude.

\section{EXPERIMENTAL SETUP}

\section{A. Filament stretching rheometry}

A filament stretching rheometer capable of imposing a homogeneous uniaxial extensional flow on a fluid filament placed between its two endplates was used to make simultaneously measurements of the evolution of the force, the midpoint radius, and the flow induced birefringence of the fluid filament. A schematic diagram of a fluid filament experiencing a homogeneous uniaxial extensional flow is shown in Fig. 1. A complete description of the filament stretching rheometer design and operating space can be found in Rothstein and McKinley (2002a), (2002b). Filament stretching rheometry has, in re- 


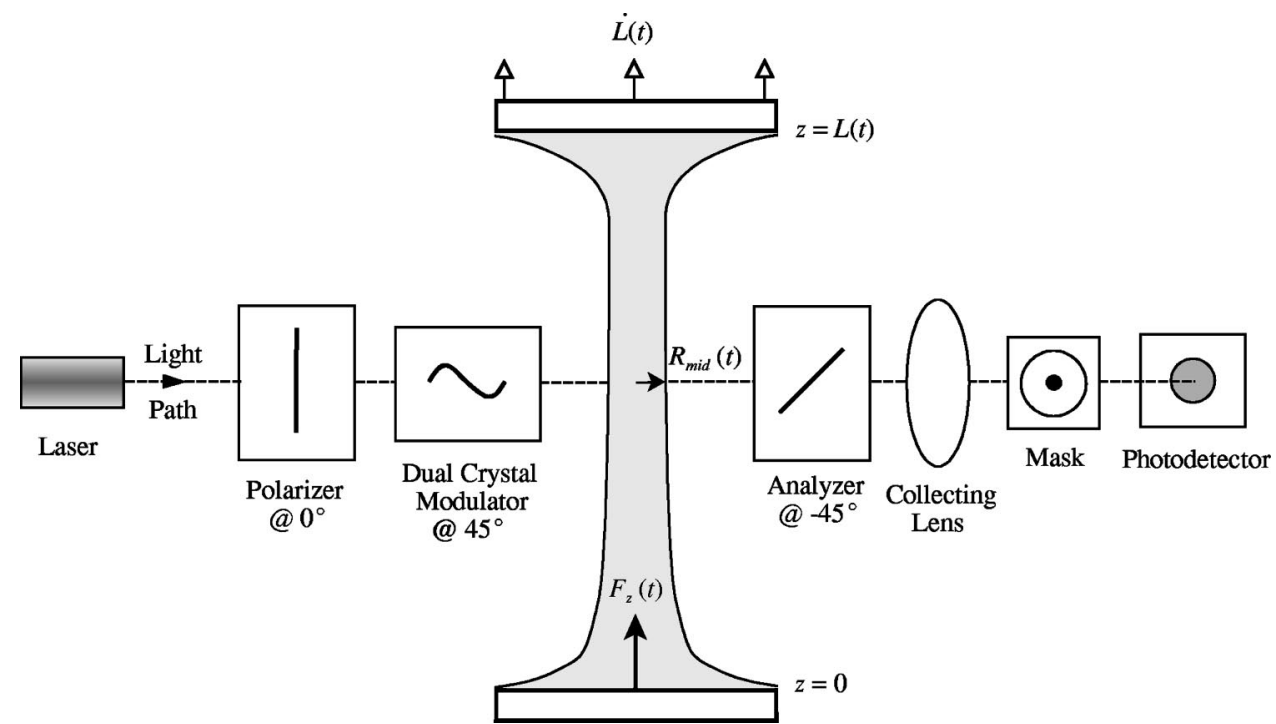

FIG. 1. Schematic diagram of fluid within a filament stretching rheometer and the flow induced birefringence optical train.

cent years, been shown to be an accurate and reproducible technique for measuring the transient extensional rheology of polymer solutions and melts [Anna et al. (2001); McKinley and Sridhar (2002)].

If the endplates of the filament stretching rheometer in Fig. 1 are separated exponentially in time, this test is classified as a type II experiment [Kolte et al. (1997)]. Type II experiments are easily implemented in a filament stretching rheometer, but result in an inhomogeneous extension rate. In order to accurately determine the extensional properties of the wormlike micelle solutions, a type III experiment must be performed such that the extension rate imposed on the fluid filament

$$
\dot{\epsilon}=-\frac{2}{R_{\text {mid }}(t)} \frac{d R_{\text {mid }}(t)}{d t}
$$

is constant. In order to achieve a constant extension rate, the motion of the endplates must be controlled so that the midpoint radius of the fluid filament, $R_{\text {mid }}(t)$, decays exponentially with time. This is achieved through a master curve inversion technique which generates the necessary type III test profiles from a type II master curve [Anna et al. (1999); McKinley et al. (2001); Orr and Sridhar (1999)].

The total deformation of the system can be described in terms of a Hencky strain

$$
\epsilon=\int_{0}^{t} \dot{\epsilon} d t^{\prime}=-2 \ln \left(R_{\mathrm{mid}} / R_{0}\right),
$$

while the strength of the extensional flow is characterized by the Deborah number which is the ratio of the characteristic relaxation time of the fluid, $\lambda$, to the characteristic timescale of the flow, $\dot{\epsilon}^{-1}$ :

$$
\mathrm{De}=\lambda \dot{\epsilon} .
$$

In a detailed force balance of the filament stretching rheometer, Szabo (1997) showed that the principal elastic tensile stress difference generated within the filament could be 
calculated from the total force measured by the load cell, $F_{z}$, if the weight of the fluid, the surface tension and the inertia of the fluid are taken into account

$$
\left\langle\tau_{z z}-\tau_{r r}\right\rangle=\frac{F_{z}}{\pi R_{\text {mid }}^{2}}+\frac{1}{2} \frac{\rho g\left(\pi L_{0} R_{0}^{2}\right)}{\pi R_{\text {mid }}^{2}}-\frac{\sigma}{R_{\text {mid }}}+\frac{1}{2} \frac{\rho\left(\pi L_{0} R_{0}^{2}\right) \ddot{L}_{z}}{\pi R_{\text {mid }}^{2}} .
$$

In the earlier equation, $\sigma$ is the surface tension of the fluid, $\rho$ is the density of the fluid, and $\ddot{L}_{z}$ is the acceleration of the endplate. For strain hardening fluids, the contributions of surface tension and gravity to the force balance in Eq. (4) are only significant at small strains or strain rates [Anna et al. (2001)]. The last term of Eq. (4) is due to the fluid inertia and is negligibly small for the present experiments. The principal elastic tensile stress can be further broken down into contributions from the wormlike micelle and its Newtonian solvent

$$
\left\langle\tau_{z z}-\tau_{r r}\right\rangle=\Delta \tau_{e}+3 \eta_{s} \dot{\epsilon}
$$

where $\eta_{s}$ is the solvent viscosity of the fluid. The transient extensional viscosity of polymeric fluids in homogenous uniaxial extension can thus be written as

$$
\eta_{E}^{+}=\frac{\left\langle\tau_{z z}-\tau_{r r}\right\rangle}{\dot{\epsilon}}=3 \eta_{s}+\frac{\Delta \tau_{e}}{\dot{\epsilon}}
$$

and nondimensionalized with the zero shear rate viscosity to form the Trouton ratio is defined as $\operatorname{Tr}=\eta_{E}^{+} / \eta_{0}$.

\section{B. Flow induced birefringence}

The refractive index of a wormlike micelle is different in the directions parallel to and normal to the axis of the micelle tube. Flow induced birefringence measurements have been used quite extensively to examine steady and transient flows of wormlike micelle solutions [Fuller (1995); Hu et al. (1993); Lerouge and Decruppe (2000); Shikata et al. (1994); Wheeler et al. (1996)]. By passing light of a known polarization state and frequency through the polymeric fluid sample and measuring the resulting change in polarization state, flow induced birefringence can be used to determine the local anisotropy in the conformation of the wormlike micelles, $\Delta A=A_{11}-A_{22}$ [Fuller (1995)]. The wormlike micelle conformation is characterized by the ensemble average second moment tensor, $\mathbf{A}=\langle\mathbf{Q Q}\rangle / Q_{\text {eq }}^{2}$, which is made dimensionless by the square of the equilibrium value of the end-to-end vector of the wormlike micelle, $\mathbf{Q}$. In a homogeneous uniaxial extensional flow the resulting expression for the birefringence is:

$$
\frac{\Delta n^{\prime}}{C}=G_{N}^{0} \Delta A
$$

where $\Delta n^{\prime}$ is the measured birefringence, $C$ is the stress-optical coefficient, and $G_{N}^{0}$ is the plateau modulus of the fluid.

The optical path for the polarization modulated flow birefringence system employed in this research is shown in Fig. 1. For a complete description of the optical train design and challenges of implementation on a filament stretching rheometer see Rothstein and McKinley (2002a), (2002b). In this research, the optical train is aligned in the filament stretch direction resulting in an extinction angle that does not deviate from $\chi=0$. The retardation, thus simplifies to 


$$
\delta=\frac{4 \pi \Delta n^{\prime} R_{\mathrm{mid}}(t)}{\lambda_{\text {light }}}=\cos ^{-1}\left[ \pm \sqrt{1-\left(\frac{I_{\omega}}{2 J_{1}\left(A_{c}\right) I_{\mathrm{dc}}}\right)^{2}}\right],
$$

where $A_{c}$ is the amplitude of the electro-optical modulation calibrated such that the order zero Bessel function of the first kind is equal to $J_{0}\left(A_{c}\right)=0$ [Frattini and Fuller (1984)]. The intensity of the light measured by the photodetector is given by

$$
I(t)=I_{\mathrm{dc}}+I_{\omega} \sin \omega t+I_{2 \omega} \cos 2 \omega t
$$

For the experiments presented in this study, the minimum resolvable retardation of this system is approximately $\delta \approx 1 \times 10^{-2} \mathrm{rad}$.

\section{Description of test fluids}

The test fluids are a series of wormlike micelle solutions of a surfactant, CTAB (Fisher Scientific), and a salt, NaSal (Fisher Scientific), in de-ionized water. The concentration of the surfactant and the salt in solution were varied independently from $0.025 \leqslant \mathrm{~mol} /$ $1 \leqslant 0.10$. Each of these solutions is well above the critical micelle concentration (CMC), which for CTAB in pure water is $\mathrm{CMC}=9 \times 10^{-4} \mathrm{~mol} / 1$ [Israelachvili (1985)]. At these concentrations, the wormlike micelle solutions are concentrated and entangled with significant number of entanglement points per chain [Israelachvili (1985)]. The solutions were prepared and allowed to equilibrate at room temperature for several days before experiments were performed.

The flow induced birefringence measurements were aided by the extremely large value of the stress-optic coefficient of CTAB surfactant solutions which facilitate accurate measurements of even small changes in the anisotropy of the wormlike micelle conformation. The stress optic coefficients of a series of CTAB/NaSal solutions were measured by Shikata et al. (1994) and found to be independent of surfactant and salt concentration above the critical micelle concentration. Following the work of Shikata et al. (1994) the stress-optic coefficient of each of the solutions tested was assumed to be $C=-3.1 \times 10^{-7} \mathrm{~Pa}^{-1}$. This assumption is validated in Sec. III by the excellent agreement between the extensional rheology data at small strains and the stress-optical law.

The equilibrium surface tension for each of the wormlike micelle solutions tested was assumed to be consistent with the value of $\sigma=0.036 \mathrm{~N} / \mathrm{m}$ reported in the literature for $\mathrm{CTAB} / \mathrm{NaSal}$ solutions above the CMC [Cooper-White et al. (2002)]. However, because of the relatively short timescales associated with the filament stretching experiments, it may be the dynamic and not the equilibrium surface tension which is the relevant quantity. As the fluid filament is stretched and new surface is generated, CTAB molecules diffuse from the bulk and populate the surface. The time scale for repopulating the surface can be quite long. Using a maximum bubble pressure tensiometer Cooper-White et al. (2002) measured the dynamic surface tension of a series of CTAB/NaSal solutions. Above the CMC, the dynamics surface tension behavior was found to be independent of surfactant concentration. At short times, up to about $15 \mathrm{~ms}$ after the creation of a new surface, the dynamic surface tension equals that of water $(\sigma=0.07 \mathrm{~N} / \mathrm{m})$. As time progresses, the surface tension decays, eventually approaching an equilibrium value of $\sigma=0.036 \mathrm{~N} / \mathrm{m}$ after about $1000 \mathrm{~ms}$ [Cooper-White et al. (2002)]. It can be shown that for the rate of surface expansion experienced by a fluid filament elongated within the filament stretching rheometer, the dynamics surface tension will be at or near its equilibrium value. Any errors incurred in the calculation of elastic tensile stress by assuming the surface tension is at its equilibrium value will be negligible at all by the smallest strains. 
On the other hand, dynamic surface tension effects may play a significant role in the filament rupture dynamics which occur over much shorter times.

All extensional rheology experiments were performed at room temperature. When analyzing and presenting the experimental data, the relaxation times and viscosities were adjusted to their values at a reference temperature of $T_{0}=25^{\circ} \mathrm{C}$ using time-temperature superposition with a shift factor, $a_{T}$, defined by the Williams-Landel-Ferry equation [Bird et al. (1987)].

\section{RESULTS AND DISCUSSION}

\section{A. Shear rheology}

A Bohlin Instruments controlled stress rheometer (model CVO) was used with a $4 \mathrm{~cm}$ diameter and $1^{\circ}$ cone to characterize both the steady and the dynamic shear rheology of each of the surfactant solutions prepared. The micelle solutions were loaded and allowed to equilibrate at $T_{0}=25^{\circ} \mathrm{C}$ for several minutes. The samples were not presheared. The linear viscoelastic data for a representative selection of the surfactant solutions used in this study is presented in Fig. 2. In the regime of linear deformation imposed by small amplitude oscillatory shear flows, it has been shown that wormlike micelle solutions can be accurately modeled by a Maxwell model having just a single relaxation time [Rehage and Hoffmann (1991)]. In Fig. 2, the storage modulus, $G^{\prime}$, and loss modulus, $G^{\prime \prime}$, are plotted as a function of angular frequency, $\omega$, along with the prediction of a single mode Maxwell model. The linear viscoelastic data and the model prediction are in excellent agreement for all micelle solutions used in this study although slight deviations are observed at large angular frequencies corresponding to the Rouse-like behavior of the micelle between entanglement points [Fischer and Rehage (1997)]. These deviations, which become more pronounced as the concentration of surfactant and salt and therefore the number of entanglements per chain are reduced are consistent with observations in the literature [Berret et al. (1993)]. In Table I, the viscometric parameters characterizing the shear rheology of the wormlike micelle solutions used in this study are tabulated. The parameters represent the best fit of the linear viscoelastic data to a single-mode Maxwell model for each of the wormlike micelle solutions. The plateau moduli of the equimolar $\mathrm{CTAB}$ and NaSal wormlike micelle solutions are found to increase with increasing surfactant concentration while the relaxation times are found to decrease. For the $0.05 \mathrm{~mol} / \mathrm{l}$ $\mathrm{CTAB}$ solutions with variable salt concentration, the plateau modulus and relaxation time are again found to increase and decrease respectively with increasing salt concentration. However, above a salt concentration of $0.05 \mathrm{~mol} / \mathrm{l}$ of NaSal, very little change in the rheology is observed with further increases in the salt concentration. These results are consistent with rheological data for $\mathrm{CTAB} / \mathrm{NaSal}$ solutions found in the literature [Shikata and Kotaka (1991)].

In Fig. 3, the steady shear viscosity, $\eta$, and dynamic viscosity, $\eta^{\prime}=G^{\prime \prime} / \omega$ of the 0.05 M CTAB 0.05 M NaSal wormlike micelle solution are shown as a function of shear rate, $\dot{\gamma}$, and angular frequency, respectively. The general trends in the steady shear rheology of this wormlike micelle solution are representative of all the solutions used in this study. At small shear rates and angular frequencies, the micelle solution behaves like a rheologically simple fluid; the steady and dynamic viscosity plateau to approximately the same value. As the applied shear stress is increased, the fluid begins to shear thin. At a critical value of applied shear stress $\left(\tau_{12} \approx 20 \mathrm{~Pa}\right)$, the viscosity drops precipitously.

Superimposed over the experimental data in Fig. 3 are the predictions of several constitutive models. The solid line in Fig. 3 represent the predictions of the FENE-P model for the steady shear data. The value of the finite extensibility parameter, $L^{2}$, used 

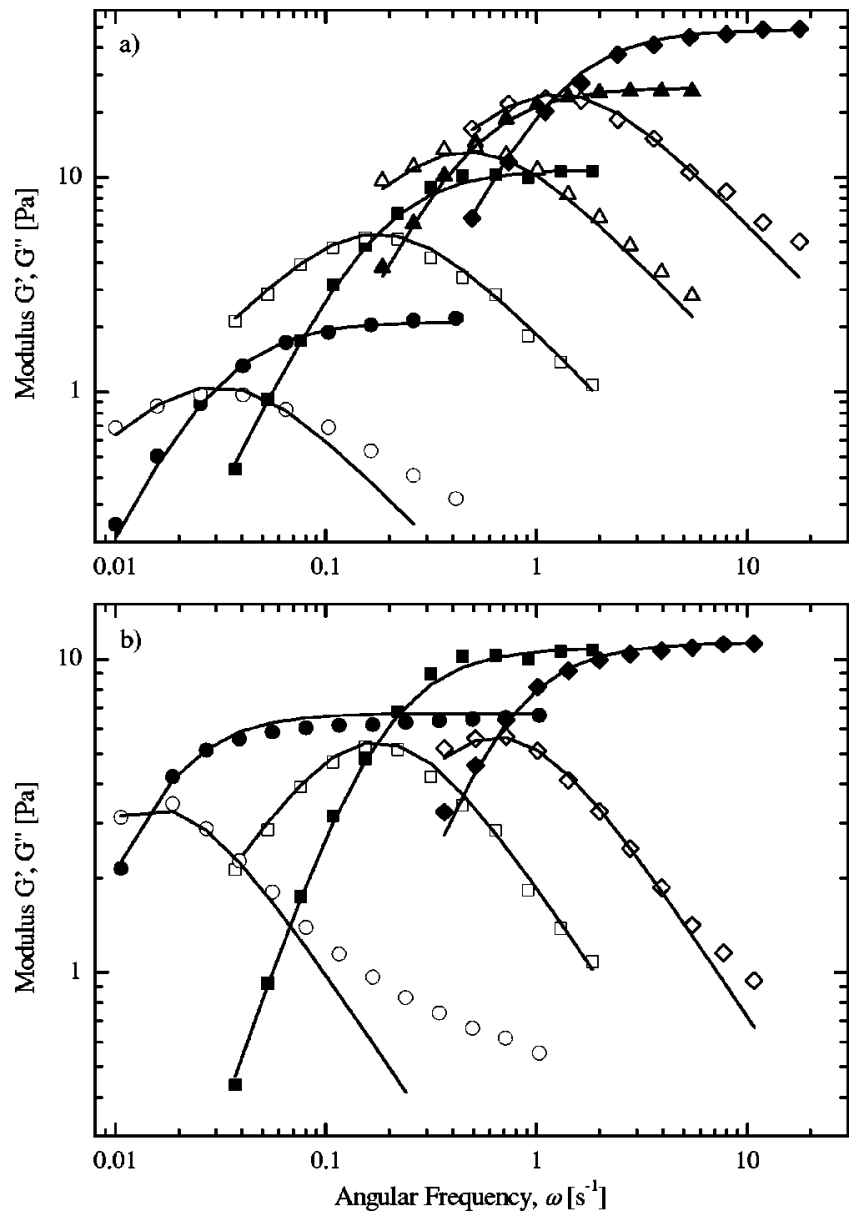

FIG. 2. Linear viscoelastic data for a series of $\mathrm{CTAB}$ and NaSal micelle solutions. The filled symbols represent the storage modulus, $G^{\prime}$, the hollow symbols represent the loss modulus, $G^{\prime \prime}$, and the solid lines represent the predictions of a single mode Maxwell model. Included in (a) are - $0.025 \mathrm{M} \mathrm{CTAB} \mathrm{0.025} \mathrm{M} \mathrm{NaSal;} \mathbf{\square}: 0.05$ M CTAB $0.05 \mathrm{M} \mathrm{NaSal}$; $: 0.075 \mathrm{M}$ CTAB $0.075 \mathrm{M} \mathrm{NaSal}$; and : 0.10 M CTAB 0.10 M NaSal. Included in (b) are : $0.05 \mathrm{M}$ CTAB $0.025 \mathrm{M} \mathrm{NaSal}$; $0.05 \mathrm{M}$ CTAB $0.05 \mathrm{M} \mathrm{NaSal}$; and : $0.05 \mathrm{M}$ CTAB $0.10 \mathrm{M}$ NaSal.

TABLE I. Parameters characterizing the shear rheology of the CTAB and NaSal wormlike micelle solutions.

\begin{tabular}{lcccc}
\hline \hline CTAB [mol/l] & NaSal $[\mathrm{mol} / \mathrm{l}]$ & Viscosity $[\mathrm{Pas}]$ & Modulus $[\mathrm{Pa}]$ & Relaxation time $[\mathrm{s}]$ \\
\hline 0.025 & 0.025 & 70 & 1.9 & 33.0 \\
0.05 & 0.05 & 62 & 10.9 & 5.7 \\
0.075 & 0.075 & 55 & 26.2 & 2.1 \\
0.1 & 0.1 & 39 & 48.8 & 0.8 \\
0.05 & 0.025 & 430 & 6.6 & 65.0 \\
0.05 & 0.075 & 16.5 & 12.7 & 1.3 \\
0.05 & 0.1 & 17.5 & 11.3 & 1.6 \\
\hline \hline
\end{tabular}




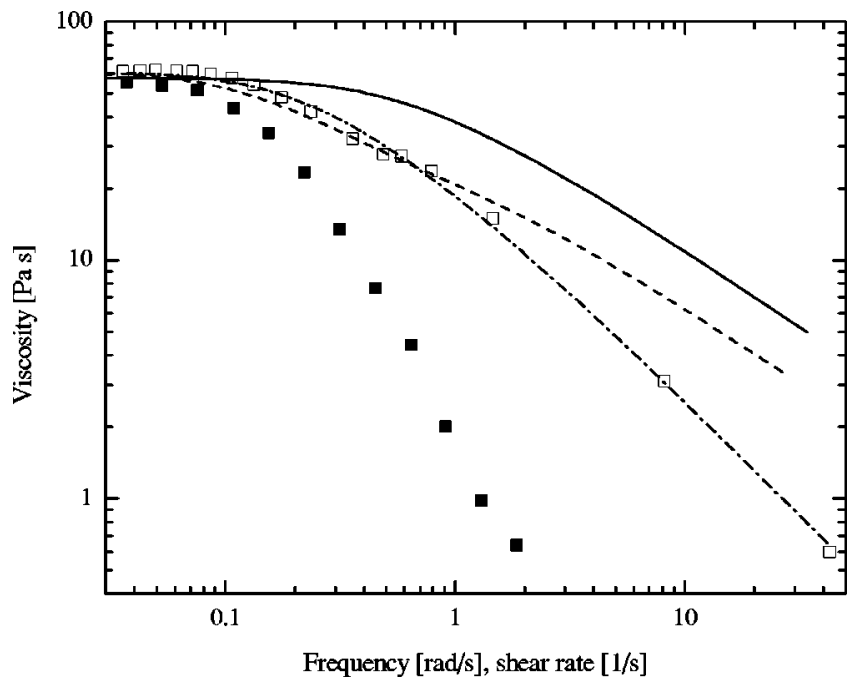

FIG. 3. Viscosity as a function of shear rate and frequency for a $0.05 \mathrm{M}$ CTAB $0.05 \mathrm{M}$ NaSal wormlike micelle solutions. Included in the figure are $\mathbf{\square}$ : the dynamic viscosity, $\eta^{\prime}=G^{\prime \prime} / \omega$; $\square$ : the viscosity, $\eta$; solid line: the FENE-P model prediction; dashed line: the Bird-DeAguiar model prediction; and dash-dot line: the Giesekus model prediction.

in the FENE-P model can be computed directly from molecular quantities, thereby avoiding the need to fit the finite extensibility parameter to the shear rheology data, a practice which has been shown to result in a systematic underprediction of the finite extensibility parameter [Rothstein and McKinley (1999)]. Shikata et al. (1994) proposed a physical model to describe wormlike micelle solutions in terms of a rubber elasticity framework. If one assumes that the wormlike micelles are Gaussian chains, then the molecular weight between entanglements, $M_{e}$, and the spacing between entanglements, $\xi$, can be approximated by Ferry (1980):

$$
\begin{gathered}
M_{e}=\frac{c N_{A} k_{B} T}{G_{N}^{0}}, \\
\xi=\left(\frac{k_{B} T}{G_{N}^{0}}\right)^{1 / 3} .
\end{gathered}
$$

In the earlier equations, $G_{N}^{0}$ is the plateau modulus determined from the linear viscoelastic data, $c$ is the micelle concentration, $N_{A}$ is Avogadro's number, $T$ is the temperature, and $k_{B}$ is the Boltzmann constant. Shitaka et al. (1994) assumed that the micelle repeat unit were in the shape of a flat disk containing $18 \mathrm{CTA}^{+} \mathrm{Sal}^{-}$complexes as shown in Fig. 4. Using light scattering measurements, they were able to determine the physical dimensions of each repeat unit. Given a disk thickness of $w=0.85 \mathrm{~nm}$, the molecular weight per unit length of wormlike micelle was determined to be $M_{L}=8900 \mathrm{~g} / \mathrm{mol} \mathrm{nm}$, independent of surfactant and salt concentration. When the wormlike micelle is fully stretched, an approximate value of the finite extensibility parameter can be calculated directly from the molecular weight between entanglements, the distance between entanglements and the molecular weight per unit length of micelle 


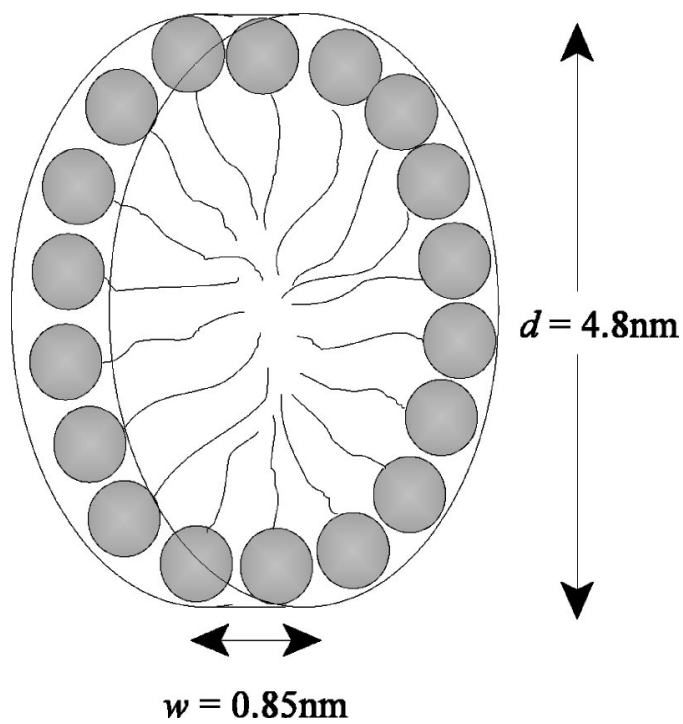

FIG. 4. Schematic of the structural model for a monomer of the wormlike micelle. The model assumes each monomer contains $18 \mathrm{CTAB}^{+} \mathrm{Sal}^{-}$complexes arranged in the shape of a flat disk.

$$
L^{2}=\left(\frac{M_{e}}{\xi M_{L}}\right)^{2}=\left(\frac{k_{B} T}{G_{N}^{0}}\right)^{4 / 3}\left(\frac{c N_{A}}{M_{L}}\right)^{2} .
$$

For the $0.05 \mathrm{M}$ CTAB $0.05 \mathrm{M} \mathrm{NaSal}$ solution, Eq. (12) suggests $L^{2}=56$. The resulting FENE-P model predictions are shown in Fig. 3. As expected, the FENE-P model does not accurately predict the onset of shear thinning in the wormlike micelle solution. However, as will be seen in Sec. III B, the FENE-P does an excellent job of predicting the micelle solution behavior in a transient homogeneous uniaxial extensional flow.

In order to achieve an improved description of the wormlike micelle solutions shear thinning behavior a more detailed treatment of hydrodynamic interactions between segments of a polymer chain undergoing a steady shearing deformation is needed. One such model that accounts for the anisotropy in the hydrodynamic drag forces in approximate form is the encapsulated dumbbell model of Bird and DeAguiar (1983) represented in Fig. 3 by the dashed line. The evolution equations of the Bird-DeAguiar model can be written as

$$
\begin{gathered}
\lambda \mathbf{A}_{(1)}=-[f(\operatorname{tr} \mathbf{A}) \mathbf{A}-\mathbf{I}]+3(1-\sigma \beta) \frac{\mathbf{A}}{\operatorname{tr} \mathbf{A}}-(1-\sigma \beta) \mathbf{I}, \\
\tau_{p}=-n k_{B} T[f(\operatorname{tr} \mathbf{A}) \mathbf{A}-\mathbf{I}]+2 n k_{B} T(1-\beta)\left[\frac{3 \mathbf{A}}{\operatorname{tr} \mathbf{A}}-\mathbf{I}\right],
\end{gathered}
$$

where

$$
f(\operatorname{tr} \mathbf{A})=\frac{1}{1-\frac{\operatorname{tr} \mathbf{A}}{L^{2}}} .
$$


The extent of anisotropy in the viscous drag on the beads is given by $\sigma$ and the anisotropy in the velocity distribution of the beads arising from Brownian motion is given by $\beta$. When $\sigma=\beta=1$, the FENE-P model is recovered. To quantitatively describe the viscometric properties of the wormlike micelle solution, the primary effect of importance is the anisotropy in the viscous drag [Rothstein and McKinley (2001)]. Setting the anisotropic drag coefficient to $\alpha=0.6$ and assuming Brownian motion of the beads to be isotropic, $\beta=1$, results in a good agreement with the steady shear data until the onset of dramatic shear thinning. The predictions of the Giesekus model [Bird et al. (1987)] with the nonlinear parameter $\alpha=0.15$ is also shown in Fig. 3. The Giesekus model is an excellent fit to the data over the entire range of shear rates.

\section{B. Transient homogenous uniaxial extensional rheometry}

A series of constant stretch rate experiments were performed on broad range of wormlike micelle solutions using the filament stretching rheometer described in Sec. II A. The spectrum of strain rates and final Hencky strains were designed to maximize the knowledge gained of the behavior of these wormlike micelle solutions in extensional flows.

Representative plots of the filament diameter, the elastic tensile stress, the average anisotropy in wormlike micelle conformation, and the Trouton ratio are presented in Fig. 5 for the $0.05 \mathrm{~mol} / \mathrm{l} \mathrm{CTAB} 0.05 \mathrm{~mol} / \mathrm{l} \mathrm{NaSal}$ solution at a Deborah number of De $=9.1$ and a final Hencky strain of $\epsilon_{r}=2.7$. In Fig. 5(a), the diameter of the fluid filament is presented as a function of time along with a solid line corresponding to an ideal type III stretch profile. A homogeneous deformation was achieved for the filament stretching experiment shown in Fig. 5(a) and subsequently for all the experiments presented in this study. The experiment came to an abrupt end at a Hencky strain of $\epsilon_{\text {rupt }}=2.7$ when the fluid filament dramatically ruptured near the axial midplane and split into two distinct domains. The dynamics of the filament rupture were too fast to be captured and analyzed quantitatively with a standard 30 frames/s charge coupled device camera, although qualitative observations were possible. Unlike capillary breakup instabilities, which has been observed to occur in weakly strain hardening fluids after considerable necking of the fluid filament [Yao et al. (1998)], the failure of these wormlike micelle filaments occurs before any significant necking has occurred at strains typically less than $\epsilon<3$. The shape of the filament remains cylindrical even at the onset of rupture. The observed filament failure mechanism appears similar to ductile failures observed in tensile loading of elastic solids and highly elastic polymer melts [Joshi and Denn (2003); Vinogradov et al. (1975)]. A surface or internal imperfection in the filament appears to develop and rapidly propagate across the filament causing a catastrophic failure of the fluid filament. A similar breakup phenomenon was first observed by Tripathi et al. (2001) during filament stretching experiments of a model associative polymer system containing hydrophobically modified ethoxylate-urathane. At high strain rates and Deborah numbers, Tripathi et al. (2001) observed the associative polymers exhibited a sudden rupture phenomenon while at low strain rates the filament was observed to fail through a viscocapillary thinning.

The growth of the elastic tensile stress difference defined in Eq. (5) is presented in Fig. 5(b). The tensile stress demonstrates considerable strain hardening and appears to approach an equilibrium value of the extensional stress around $\Delta \tau_{e} \approx 7000 \mathrm{~Pa}$. Unlike the extensional rheology of polymer solutions, no significant contribution to the stress from the aqueous solvent is observed. The response of the wormlike micelle solution is consistent with previous filament stretching experiments of concentrated and entangled polymer melts [Bhattacharjee et al. (2002); Rothstein and McKinley (2002a)]. The growth of the elastic tensile stress is well predicted by a FENE-PM model [Wedgewood et al. 

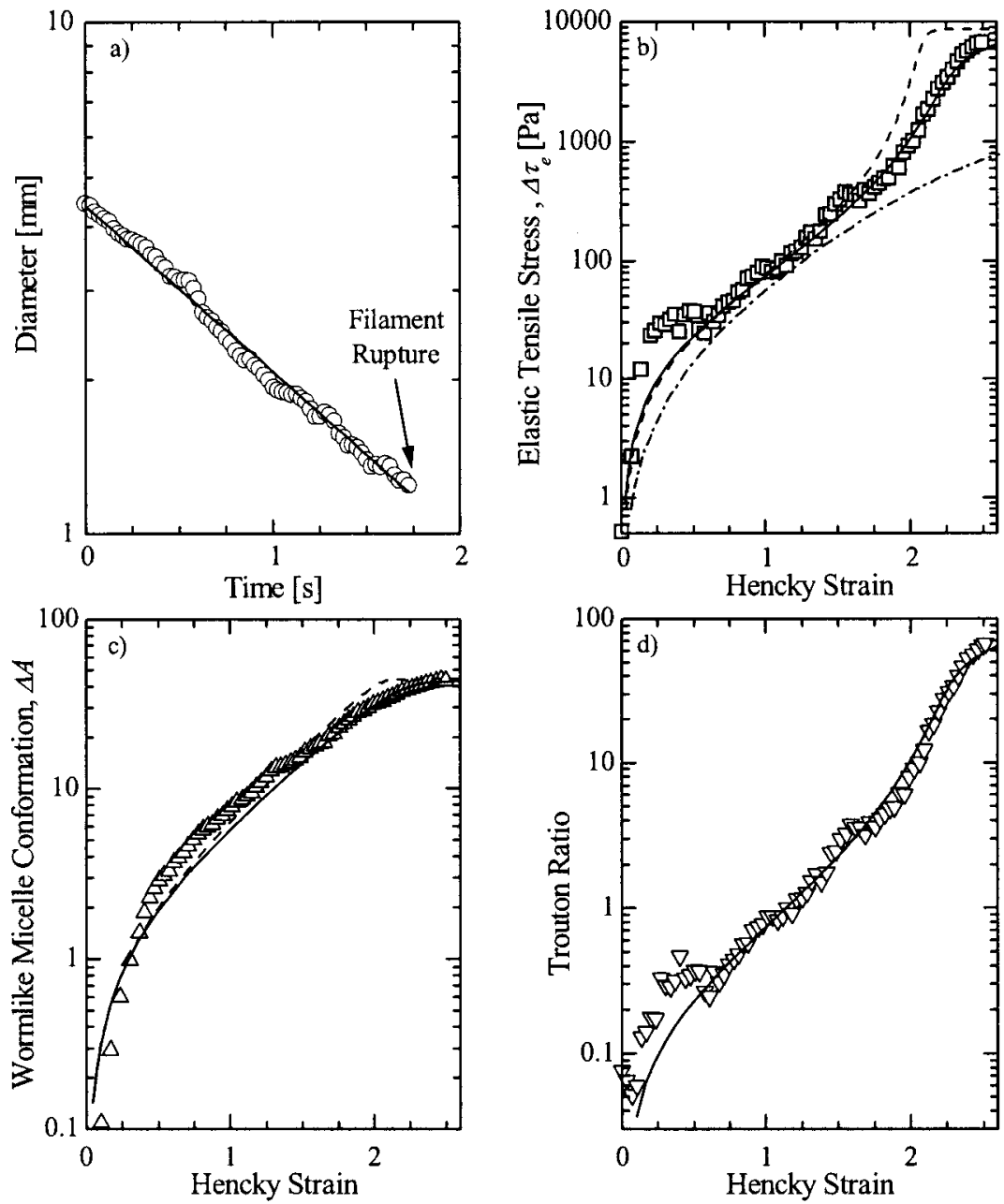

FIG. 5. Representative plots of a filament stetching experiment of a $0.05 \mathrm{M}$ CTAB $0.05 \mathrm{M}$ NaSal wormlike micelle solution at a Deborah number of $\mathrm{De}=8.6$. The experiment ends abruptly when the filament ruptures at a Hencky strain of $\epsilon_{\text {rupt }}=2.7$. Included in (a) are $\bigcirc$ : experimental measurements of filament diameter; and solid line: the ideal type III diameter profile, while (b) contains $\square$ : the elastic contribution to the polymeric tensile stress difference, (c) contains $\triangle$ : the average anisotropy in the wormlike micelle conformation, and (d) contains $\nabla$ : the Trouton ratio. Also included in (b), (c), and (d) are the predictions of solid lines: the FENE-PM model; dashed line: the Bird-DeAguiar model; and dash-dot line: the Giesekus model.

(1991)] incorporating just the first two modes of the Rouse-Zimm relaxation time spectrum. In order to achieve such an excellent agreement, the finite extensibility parameter of the FENE-PM model, $L^{2}$, was fit to the extensional data. The resulting value of $L^{2}$ $=47$ is within $20 \%$ of the value of $L^{2}=55$ predicted by the approximate theory of Shikata et al. (1994). The data are less well fit by a Bird-DeAguiar model, which, because we assumed that the Brownian motion of the beads is isotropic by setting $\beta=1$, is equivalent to a single-mode FENE-P model. The Bird-DeAguiar model strain hardens too quickly, while the Giesekus model significantly underpredicts the growth in the elastic tensile stress.

The elastic tensile stress can be rewritten as a transient extensional viscosity, Eq. (6), and nondimensionalized with the zero-shear-rate viscosity to form the Trouton ratio. The 
Trouton ration is shown as a function of Hencky strain in Fig. 5(d). The fluid demonstrates considerable strain hardening and the Trouton ratio approaches an equilibrium value of $\operatorname{Tr} \approx 80$. Because the solvent viscosity in all the wormlike micelle solutions tested are negligibly small, the Trouton ratio and the elastic tensile stress essentially differ only by a constant. Consequently, only the elastic tensile stress difference will be reported in subsequent figures.

The average anisotropy in wormlike micelle conformation defined by Eq. (7) is shown as a function of Hencky strain in Fig. 5(c). The birefringence signal is initially quite weak, but grows rapidly at short times. As time progresses and the accumulated Hencky strain increases, the anisotropy in the wormlike micelle conformation increases exponentially with time, eventually approaching an equilibrium value of $\Delta A \approx 70$. The agreement between the growth in the anisotropy in the wormlike micelle conformation and the FENE-PM model predictions is quite good. However, once again the fit of the BirdDeAguiar model overpredicts the growth in the conformation.

Even at moderate Hencky strains, all the wormlike micelle solutions that were tested showed significant strain hardening. One of the challenges associated with filament stretching experiments of wormlike micelle solutions is preventing an adhesive failure of the fluid filament at the endplates [Crosby et al. (2000); Mastrangelo (1993a), (1993b)]. To improve the adhesion of the wormlike micelle solutions to the endplates, a series of small ( 3 and $4 \mathrm{~mm}$ diameter) endplates were machined with surfaces that were modified to incorporate a pattern of $0.1 \mathrm{~mm}$ high concentric circular ridges and a knife's edge to pin the fluid contact line. These modifications successfully improved the adhesion without significantly affecting the ideality of the extensional flow at the axial midplane. Unfortunately, even with these modifications, over one half of the filament stretching experiments resulted in an adhesive failure at the endplate. These experiments were discarded and only the experiments resulting in filament rupture are included in this manuscript.

Filament stretching experiments were performed on a series of equimolar wormlike micelle solutions of CTAB and NaSal with concentrations of $0.025,0.05,0.075$, and 0.10 $\mathrm{mol} / \mathrm{l}$ as well as a series of solutions where the concentration of CTAB was held fixed at $0.05 \mathrm{~mol} / \mathrm{l}$ and the concentration of NaSal was set to $0.025,0.05,0.075$, and $0.10 \mathrm{~mol} / \mathrm{l}$. The experiments were performed over a wide range of Deborah numbers and final Hencky strains. A representative plot of the anisotropy in wormlike micelle conformation and the elastic tensile stress difference for the equimolar and variable salt concentration filament stretching experiments are shown in Figs. 6 and 7, respectively. In each case, the finite extensibility parameter was determined by fitting a FENE-PM model prediction to the extensional data. The resulting FENE-PM model predictions are superimposed over data in Figs. 6 and 7. With the exception of the $0.05 \mathrm{~mol} / \mathrm{l} \mathrm{CTAB} 0.025 \mathrm{~mol} / \mathrm{l} \mathrm{NaSal}$ solutions which required the use of over 20 relaxation modes, the predictions of the FENE-PM model are in quantitative agreement with the experimental data using no more than three relaxation modes of the Rouse-Zimm spectrum. A complete list of the finite extensibility parameters predicted by the approximate theory of Shikata et al. (1991) and the values achieved through the best fit to the extensional data can be found in Table II. The two values of the finite extensibility parameter are found to generally be in good agreement, differing by less than $20 \%$ in each case.

It has been shown that the stress-optical rule is valid for entangled systems provided that there is only deformation of the constraining tubes and no stretching of the chain contained within the tubes [Ianniruberto and Marrucci (1998)]. Based on reptation theory, at high Deborah numbers, chain stretching can lead to a nonlinear relationship between the polymer stress and flow-induced birefringence and a breakdown of the stress-optical 


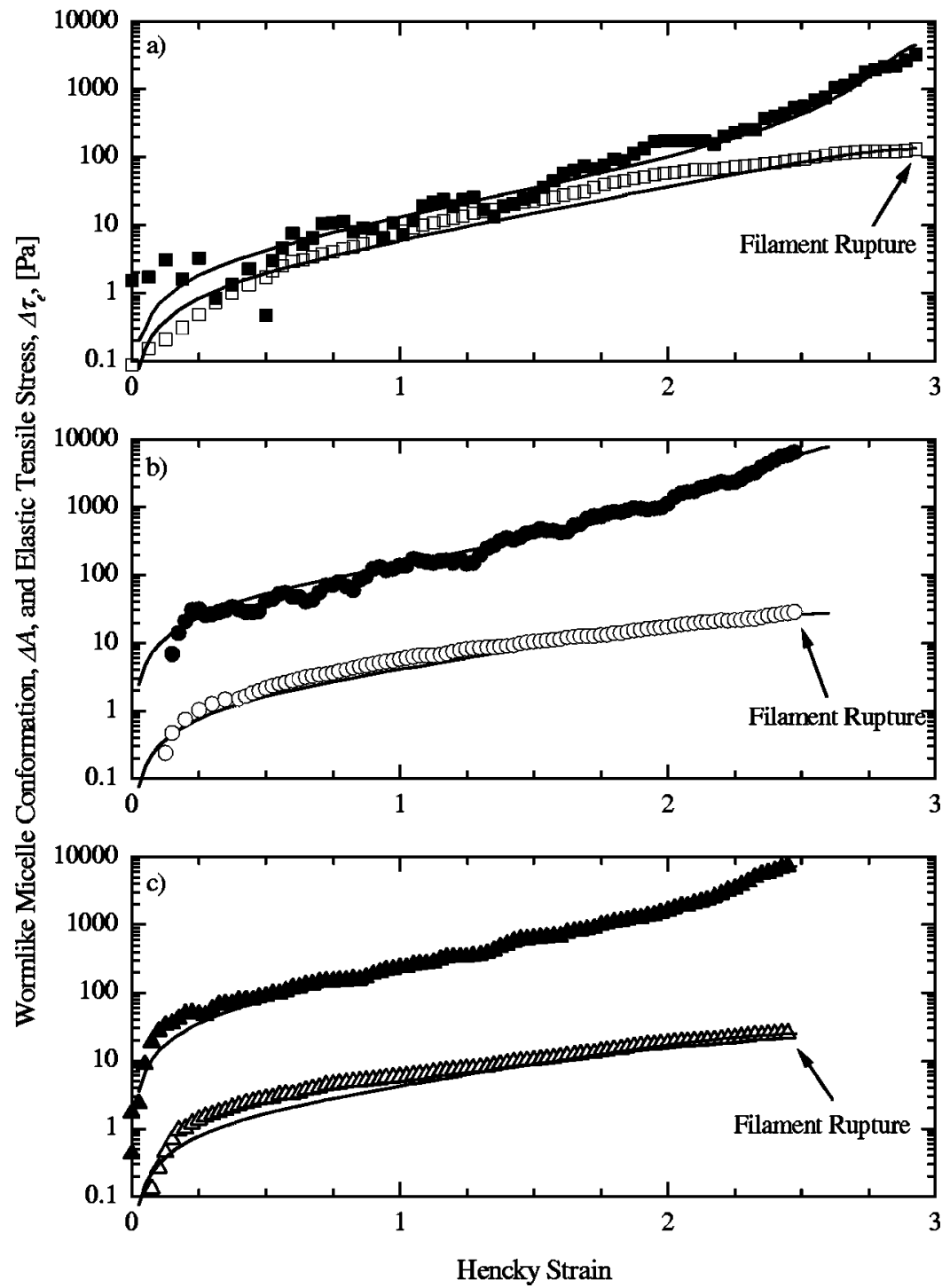

FIG. 6. Transient filament stretching rheometry experiments of a series of equimolar wormlike micelle solutions of CTAB and NaSal. The filled symbols represent the elastic contribution to the tensile stress difference, the hollow symbols represent the average anisotropy in the wormlike micelle conformation and the solid lines are the predictions of the FENE-PM model. Included in (a) are $\mathbf{\square}$ : and $\square$ : for a 0.025 M CTAB 0.025 M NaSal wormlike micelle solutions at $\mathrm{De}=49.5$. Included in (b) are $\bullet$ : and $\bigcirc$ : for a $0.075 \mathrm{M} \mathrm{CTAB} 0.075 \mathrm{M} \mathrm{NaSal}$ wormlike micelle solutions at $\mathrm{De}=3.2$. Included in (c) are $\boldsymbol{\Delta}$ : and $\triangle$ : for a $0.10 \mathrm{M} \mathrm{CTAB} 0.10 \mathrm{M} \mathrm{NaSal}$ wormlike micelle solutions at $\mathrm{De}=2.4$. In each figure, the experiment ends abruptly upon the rupture of the fluid filament.

law [Venerus et al. (1999)]. Significant strain-hardening and thus chain stretching is observed for all the fluids tested. As the salt and surfactant concentration is increased, the wormlike micelle solutions approach and then realize a steady-state value of the elastic tensile stress and average anisotropy in the wormlike micelle conformation. For the experiments presented in Figs. 5, 6, and 7, the stress-optical law is found to be violated 


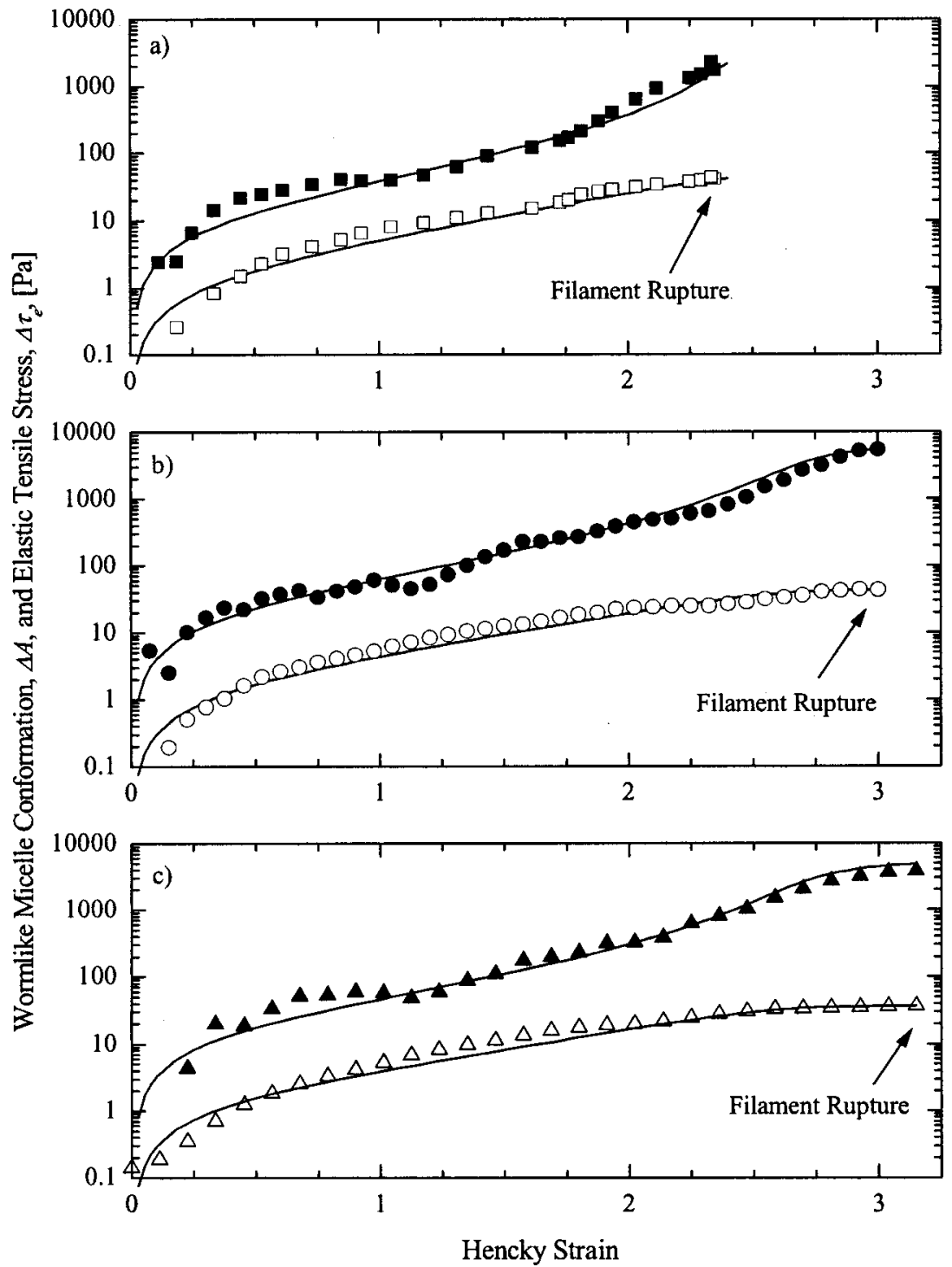

FIG. 7. Transient filament stretching rheometry experiments for series of wormlike micelle solutions holding the concentration of CTAB fixed while varying the concentration of NaSal. The filled symbols represent the elastic contribution to the tensile stress difference, the hollow symbols represent the average anisotropy in the wormlike micelle conformation and the solid lines are the predictions of the FENE-PM model. Included in (a) are $\square$ : and $\square$ : for a $0.05 \mathrm{M}$ CTAB $0.025 \mathrm{M} \mathrm{NaSal}$ wormlike micelle solutions at De $=1125$. Included in (b) are -: and $\bigcirc$ : for a $0.05 \mathrm{M}$ CTAB $0.075 \mathrm{M} \mathrm{NaSal}$ wormlike micelle solutions at De $=3$.9. Included in (c) are $\mathbf{\Delta}$ : and $\triangle$ : for a $0.05 \mathrm{M} \mathrm{CTAB} 0.10 \mathrm{M} \mathrm{NaSal}$ wormlike micelle solutions at De $=7.0$. In each figure, the experiment ends abruptly upon the rupture of the fluid filament.

at elastic tensile stress differences between $200 \mathrm{~Pa}<\Delta \tau_{e}<1000 \mathrm{~Pa}$, depending on surfactant and salt concentration.

The filament stretching experiments shown in Figs. 6 and 7 all end with the rupture of the fluid filament near the axial midplane. Average values of the maximum elastic tensile stress achieved before rupture, $\Delta \tau_{\text {rupt }}$, and the maximum anisotropy in the end-to-end micelle conformation, $\Delta A_{\text {rupt }}$, were calculated for all the filament stretching experiments 
TABLE II. Parameters characterizing the extensional rheology of the CTAB and NaSal wormlike micelle solutions and the failure of the fluid filament.

\begin{tabular}{llccrrr}
\hline \hline $\begin{array}{l}\text { CTAB } \\
{[\mathrm{mol} / 1]}\end{array}$ & $\begin{array}{c}\text { NaSal } \\
{[\mathrm{mol} / \mathrm{l}]}\end{array}$ & $\begin{array}{c}\text { Theoretical } \\
\text { extensibility } \\
\text { parameter, } b=L^{2}\end{array}$ & $\begin{array}{c}L^{2} \text { from } \\
\text { FENE-PM } \\
\text { model fit }\end{array}$ & $\Delta \tau_{\text {rupt }} / G_{N}^{0}$ & $\Delta A_{\text {rupt }}$ & $E_{\text {sciss }} / k_{B} T$ \\
\hline 0.025 & 0.025 & 142 & 144 & 1015 & 102 & 3.8 \\
0.05 & 0.05 & 56 & 47 & 460 & 43 & 4.3 \\
0.075 & 0.075 & 38 & 32 & 279 & 31 & 4.1 \\
0.1 & 0.1 & 30 & 30 & 205 & 26 & 4.1 \\
0.05 & 0.025 & 94 & 75 & 538 & 54 & 3.6 \\
0.05 & 0.075 & 45 & 48 & 356 & 40 & 4.0 \\
0.05 & 0.1 & 53 & 42 & 336 & 39 & 3.5 \\
\hline \hline
\end{tabular}

for each micelle solution that ended in the rupture of the fluid filament. In each case, the rupture of the fluid filament occurs at more than $85 \%$ of its finite extensibility limit. For the equimolar solutions shown in Figs. 5 and 6, $\Delta \tau_{\text {rupt }}$ increases while $\Delta A_{\text {rupt }}$ decreases with increasing micelle concentration. When the concentration of surfactant is held fixed and the salt concentration is varied, as in Figs. 5 and 7, $\Delta \tau_{\text {rupt }}$ is found to be a nonmonotonic function of the salt concentration. If, however, the differences in the elasticity of the wormlike micelle solutions are accounted for by nondimensionalizing the maximum tensile stress achieved before rupture of each fluid with the plateau modulus, $\Delta \tau_{\text {rupt }} / G_{N}^{0}$ and $\Delta A_{\text {rupt }}$ are found to decrease monotonically with increasing micelle concentration and salt content. Values of, $\Delta \tau_{\text {rupt }} / G_{N}^{0}$ and $\Delta A_{\text {rupt }}$ are compiled in Table II. The dimensionless tensile stress is found to be a strong function of micelle concentration for equimolar solutions, decreasing roughly as $\Delta \tau_{\text {rupt }} / G_{N}^{0} \sim c_{\text {CTAB }}^{-1}$, while for a given surfactant concentration the dependence on salt concentration is significantly weaker, decreasing roughly as $\Delta \tau_{\text {rupt }} / G_{N}^{0} \sim c_{\mathrm{NaSal}}^{-1 / 3}$.

To compare the results from our filament stretching experiments to the opposed jet devise experiments of wormlike micelle solutions in the literature [Chen and Warr (1997); Fischer et al. (1997); Prud'homme and Warr (1994); Walker et al. (1996)], the maximum value of the extensional viscosity achieved before filament rupture, $\eta_{E}$, is plotted as a function of extension rate, $\dot{\epsilon}$, in Fig. 8. All the extension rates presented in Fig. 8 are large enough to result in the coil-stretch transition, De $\gg 0.5$. For all the wormlike micelle solutions and extension rates tested, the extensional viscosity was found to be significantly larger than the Newtonian limit of $3 \eta_{0}$ and thus the resulting Trouton ratio are uniformly found to be $\operatorname{Tr} \gg 3$. The extensional viscosity is found to decay monotonically with increasing extension rate. This result is consistent with the results observed at high extension rates from extensional viscosity measurements obtained with opposed jet devices [Chen and Warr (1997); Prud'homme and Warr (1994); Walker et al. (1996)]. In their experiments, the extensional viscosity was found to first increase from the Newtonian limit and then, at a critical extension rate, decrease with increasing extension rate. Unfortunately, the lower extension rate data could not be reproduced in the filament stretching rheometer because of problems arising from gravitational sagging and capillary draining of the fluid filament [Anna and McKinley (2001); Anna et al. (2001)].

Prud'homme and Warr (1994) theorized that the observed reduction in the extensional viscosity at high extension rates was the result of a scission of the wormlike micelles in the strong extensional flow. The hypothesis of Prud'homme and Warr was later supported by light scattering measurements [Chen and Warr (1997)] which demonstrated a decrease 


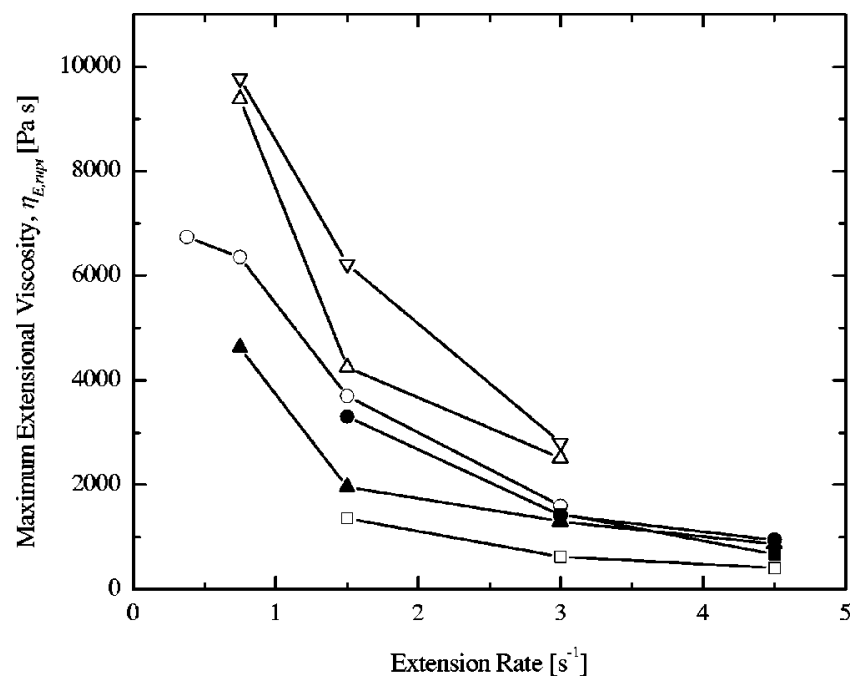

FIG. 8. Maximum extensional viscosity achieved before filament rupture as a function of extension rate for a series of CTAB and NaSal micellar solutions. The data includes $\square: 0.025 \mathrm{M}$ CTAB $0.025 \mathrm{M} \mathrm{NaSal}$; $\bigcirc: 0.05 \mathrm{M}$ CTAB $0.05 \mathrm{M} \mathrm{NaSal} ; \triangle: 0.075 \mathrm{M}$ CTAB $0.075 \mathrm{M} \mathrm{NaSal} ; \nabla: 0.10 \mathrm{M}$ CTAB $0.10 \mathrm{M} \mathrm{NaSal}$; $\mathbf{\square}: 0.05 \mathrm{M}$ CTAB $0.025 \mathrm{M} \mathrm{NaSal}$; : $0.05 \mathrm{M}$ CTAB 0.05 M NaSal; and $\mathbf{\Delta}: 0.05 \mathrm{M}$ CTAB 0.10 M NaSal.

in micelle radius of gyration coinciding with the onset of extensional viscosity thinning. Our observations lend further support to Prud'homme and Warr's hypothesis. Within the standard deviation of the data, our experiments suggest that the filaments of each wormlike micelle solution ruptured at a critical value of elastic tensile stress difference, nearly independent of extension rate. The resulting extensional viscosity of the wormlike micelle solutions presented in Fig. 8 thus decays as $\eta_{E \text {,rupt }} \propto \dot{\epsilon}^{-1}$. These results are in qualitative agreement with the results of Walker et al. (1996) who showed a similar dependence of extensional viscosity at large extension rates for a series of cetylpyridinium chloride $(\mathrm{CPCl}) / \mathrm{NaCl}$ wormlike surfactant solutions tested in an opposed jet device.

The presence of a critical stress for filament rupture which is independent of extension rate suggests that the rupture might be a macroscopic manifestation of a scission of the wormlike micelles within the fluid filament. As the filament is stretched, the micelles align with the flow direction and the elongate. As can be seen in Table II, at the point of rupture, the wormlike micelles have been elongated to between $84 \%$ and $98 \%$ of their finite extensibility limit. At this point, the stress exerted on an individual micelle is significant. In a solution at equilibrium, the micelles are constantly breaking and reforming with a characteristic breaking time of $\lambda_{\text {break }}$. Cates (1987) showed that the characteristic breaking time was inversely proportional to the length of the micelle. Unlike a polymer which requires energies on the order of 100's of $k_{B} T$ to result in the scission of the macromolecule [Odell et al. (1988)], the scission energy of a wormlike micelle is expected to only require "a few" $k_{B} T$ [Turner and Cates (1992)].

It is possible to approximate the scission energy of a wormlike micelle from our measurements of filament rupture. Assuming the wormlike micelles are fully extended, they can be approximated as rigid rods. The number of micelles per unit area supporting the elastic tensile stress can thus be calculated directly from the concentration of micelles. The force per micelle, $F$, can then calculated by dividing the tensile stress at rupture by the micelles per unit area. It is assumed that to scission the micelle this tensile force must act over a distance approximately equal to the micelle diameter which from Shikata et al. 
(1994) is assumed to be $D=4.8 \mathrm{~nm}$. The result is an approximate scission energy of $E_{\text {sciss }}=F D$. The scission energy was calculated for all the wormlike micelle solutions tested and is listed in Table II. The scission energy is found to be nearly independent of surfactant or salt concentration $E_{\text {sciss }} / k_{B} T \approx 3.9 \pm 0.3$. This suggests that the underlying mechanism of the filament rupture mechanism is directly related to the maximum tensile stress that can be supported by an individual elongated wormlike micelle chain. It is possible that the filament rupture is the result of a stress-induced morphology change within the fluid [Kadoma and van Egmond (1998); Liu and Pine (1996)]. However, the lack of supporting evidence such as change in the turbidity of the fluid filament or a dramatic change in the birefringence signal suggest otherwise. A more complete understanding of the failure mechanism in a pure extensional flow is still needed in order to better understand more complicated flows of a wormlike micelle solutions. An example is the flow past a sedimenting sphere for which it has recently been speculated that a similar failure of the wormlike micelles in the strong extensional flows in the wake of the sphere can lead to the onset of a newly observed flow instability [Chen and Rothstein (2002)].

A recently discovered feature of strong extensional flows of dilute polymer solutions is the presence of a stress-conformation hysteresis [Doyle et al. (1998); Rothstein and McKinley (2002b)]. Simultaneous measurements of the elastic tensile stress and average anisotropy in the polymer conformation during uniaxial elongation of dilute polymer solutions has shown that the stress evolves along different paths during stretching and the subsequent free relaxation. Filament stretching experiments on semidilute and concentrated entangle polymer solution and melts have failed to demonstrate a stressconformation hysteresis [Rothstein and McKinley (2002a)] because the extension rates were not fast enough to generate the necessary 'kinked' chain configurations between entanglement points known to result in stress-conformation hysteresis [Perkins et al. (1997)].

A kinked wormlike micelle is a nonequilibrium configuration resulting in a large local stress concentration at the kink. Since the micelle is capable of breaking and reforming, it is unlikely that these kinked conformations would survive long enough to result in a significant stress-conformation hysteresis. In Fig. 9, the elastic tensile stress difference is plotted as a function of the average anisotropy in the wormlike micelle conformation for two representative solutions, the $0.05 \mathrm{~mol} / \mathrm{l} \mathrm{CTAB} 0.05 \mathrm{~mol} / \mathrm{l} \mathrm{NaSal}$ solution and the 0.10 $\mathrm{mol} / \mathrm{l} \mathrm{CTAB} 0.10 \mathrm{~mol} / \mathrm{l} \mathrm{NaSal}$ solution, at Deborah numbers of $\mathrm{De}=9.1$ and 5.3, respectively. As anticipated, neither wormlike micelle solution demonstrates a stressconformation hysteresis even though significant chain stretching and strain hardening are observed to result in a breakdown of the stress-optical law. In fact, none of the solutions tested demonstrated a stress-conformation hysteresis.

\section{CONCLUSIONS}

In this work, a filament stretching rheometer was used to follow the evolution in the tensile force and the flow induced birefringence of a series of well characterized CTAB/ NaSal wormlike micelle solutions in an transient uniaxial elongational flow. The linear viscoelastic shear rheology of the wormlike micelle solutions was demonstrated to be well described by an upper convected Maxwell model with a single relaxation time. In extension, the wormlike micelle solutions demonstrated significant strain hardening and a failure of the stress-optical law at very low stresses, although, no stress-conformation hysteresis was observed. A quantitative fit to the transient extensional rheology of each wormlike micelle solutions was achieved using a FENE-PM model with as few as two relaxation modes. At a critical stress, nearly independent of strain rate, the wormlike 


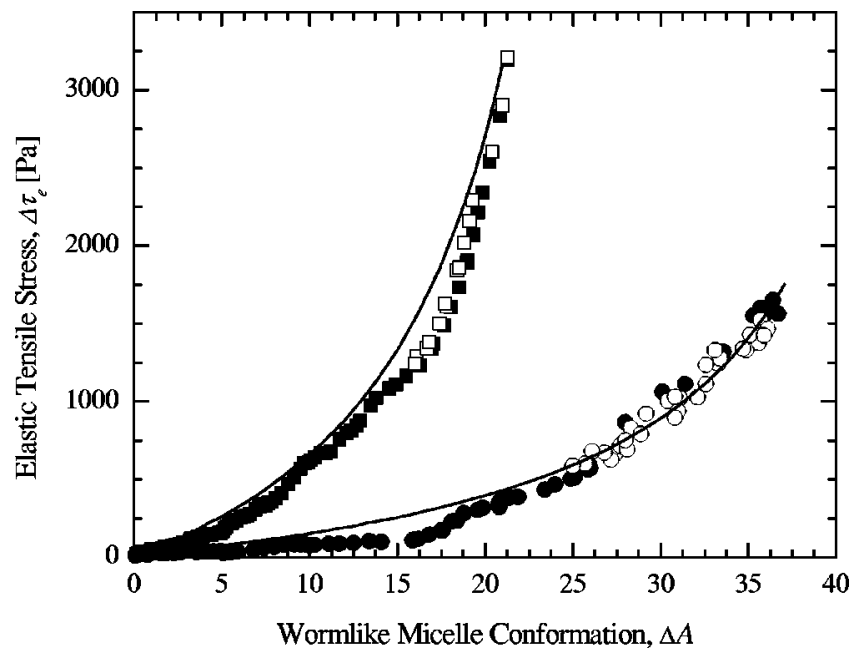

FIG. 9. Elastic tensile stress difference as a function of average wormlike micelle conformation for a filament stretching experiment. Included in the data are filled symbols representing measurements taken during stretch and hollow symbols representing measurements taken after cessation of stretch for $\mathbf{m}$ : a $0.10 \mathrm{M} \mathrm{CTAB} 0.10 \mathrm{M}$ $\mathrm{NaSal}$ wormlike micelle solution at $\mathrm{De}=5.3$ and to a final Hencky strain of $\epsilon_{f}=2.2$; $\mathbf{0}$ : a $0.05 \mathrm{M} \mathrm{CTAB} 0.05$ $\mathrm{M} \mathrm{NaSal}$ wormlike micelle solution at $\mathrm{De}=9.1$ to $\epsilon_{f}=2.3$; and solid line: the FENE-PM model predictions.

micelle solutions failed through a dramatic rupture of the fluid filaments at the axial midplane. It is conjectured that the filament failure might stem from the local scission of wormlike micelles. An analysis is performed that demonstrates the energy of wormlike micelle chain scission to be roughly $4 k_{B} T$ for all the solutions tested, independent of surfactant or salt concentration and extension rate.

\section{ACKNOWLEDGMENTS}

The author would like to thank Gareth H. McKinley of the Mechanical Engineering Department of MIT for use of his filament stretching rheometer and David Hoagland of the Polymer Science Department of the University of Massachusetts for the use of his shear rheometer.

\section{References}

Anna, S. L., and G. H. McKinley, "Elasto-capillary thinning and breakup of model elastic liquids," J. Rheol. 45, 115-138 (2001).

Anna, S. L., G. H. McKinley, D. A. Nguyen, T. Sridhar, S. J. Muller, J. Huang, and D. F. James, “An inter-laboratory comparison of measurements from filament stretching rheometers using common test fluids," J. Rheol. 45, 83-114 (2001).

Anna, S. L., C. B. Rogers, and G. H. McKinley, "On controlling the kinematics of a filament stretching rheometer using a real-time active control mechanism," J. Non-Newtonian Fluid Mech. 87, 307-335 (1999).

Belmonte, A., "Self-oscillations of a cusped bubble rising through a micellar solution," Rheol. Acta 39, 554559 (2000).

Berret, J.-F., J. Appell, and G. Porte, "Linear rheology of entangled wormlike micelles," Langmuir 9, 28512854 (1993).

Berret, J.-F., D. C. Roux, and P. Lindner, "Structure and rheology of concentrated wormlike micelles at the shear-induced isotropic-nematic transition,” Eur. Phys. J. B 5, 67-77 (1998). 
Bhattacharjee, P. K., J. Oberhauser, G. H. McKinley, L. G. Leal, and T. Sridhar, "Extensional rheometry of entangled solutions," Macromolecules 35, 10131-10148 (2002).

Bird, R. B., R. C. Armstrong, and O. Hassager, Dynamics of Polymeric Liquids: Volume 1 Fluid Mechanics (Wiley, New York, 1987).

Bird, R. B., and J. R. DeAguiar, "An encapsulated dumbell model for concentrated polymer solutions and melts. I. Theoretical development and constitutive equation," J. Non-Newtonian Fluid Mech. 13, 149-160 (1983).

Cates, M. E., "Reptation of living polymers: Dynamics of entangle polymers in the presence of reversible chain-scission reactions," Macromolecules 20, 2289-2296 (1987).

Cates, M. E., and M. S. Turner, "Flow-induced gelation of rodlike micelles," Europhys. Lett. 11, 681-686 (1990).

Chen, C., and G. G. Warr, "Light scattering from wormlike micelles in an elongational flow," Langmuir 13, 1374-1376 (1997).

Chen, S., and J. P. Rothstein, "Flow of a wormlike micelle past a falling sphere," J. Non-Newtonian Fluid Mech. (accepted for publication).

Cooper-White, J. J., R. Crooks, and D. V. Boger, "A drop impact study of worm-like viscoelastic surfactant solutions," Colloids Surf., A 210, 105-123 (2002).

Crosby, A. J., K. R. Shull, H. Lakrout, and C. Creton, "Deformation and failure modes of adhesively bonded elastic layers," J. Appl. Phys. 88, 2956-2966 (2000).

Doyle, P. S., E. S. G. Shaqfeh, G. H. McKinley, and S. H. Spiegelberg, "Relaxation of dilute polymer solutions following extensional flow," J. Non-Newtonian Fluid Mech. 76, 79-110 (1998).

Ferry, J. D., Viscoelastic Properties of Polymers (Wiley-Interscience, New York, 1980).

Fischer, P., G. G. Fuller, and Z. Lin, "Branched viscoelastic surfactant solutions and their responses to elongational flow," Rheol. Acta 36, 632-638 (1997).

Fischer, P., and H. Rehage, "Rheological master curves of viscoelastic surfactant solutions by varying the solvent viscosity and temperature," Langmuir 13, 7012-7020 (1997).

Frattini, P. L., and G. G. Fuller, “A note on phase-modulated flow birefringence,” J. Rheol. 28, 61-70 (1984).

Fuller, G. G., Optical Rheometry of Complex Fluids (Oxford University Press, New York, 1995).

Hu, Y., P. Boltenhagen, E. Matthys, and D. J. Pine, "Shear thickening in low-concentration solutions of wormlike micelles. II. Slip, fracture, and stability of the shear-induced phase,” J. Rheol. 42, 1209-1226 (1998).

Hu, Y., S. Q. Wang, and A. M. Jamieson, "Rheological and flow birefringence studies of a shear-thickening complex fluid-A surfactant model system," J. Rheol. 37, 531-546 (1993).

Ianniruberto, G., and G. Marrucci, "Stress tensor and stress-optical law in entangled polymers," J. NonNewtonian Fluid Mech. 79, 225-234 (1998).

Israelachvili, J. N., Intermolecular and Surface Forces: With Applications to Colloidal and Biological Systems (Academic, London, 1985).

Joshi, Y. M., and M. M. Denn, "Rupture of entangled polymeric liquids in elongational flows," J. Rheol. 47, 291-298 (2003).

Kadoma, I. A., and J. W. van Egmond, "Flow-induced nematic string phase in semidilute wormlike micelle solutions," Phys. Rev. Lett. 80, 5679-5682 (1998).

Kolte, M. I., H. K. Rasmussen, and O. Hassager, "Transient filament stretching rheometry II: Numerical simulation," Rheol. Acta 36, 285-302 (1997).

Larson, R. G., "Flow-induced mixing, demixing, and phase-transitions in polymeric fluids," Rheol. Acta 31, 497-520 (1992).

Larson, R. G., The Structure and Rheology of Complex Fluids (Oxford University Press, New York, 1999).

Lerouge, S., and J. P. Decruppe, "Correlations between rheological and optical properties of a micellar solution under shear banding flow," Langmuir 16, 6464-6474 (2000).

Liu, C. H., and D. J. Pine, "Shear-induced gelation and fracture in micellar solutions," Phys. Rev. Lett. 77, 2121-2124 (1996).

Lu, B., X. Li, L. E. Scriven, H. T. Davis, Y. Talmon, and J. L. Zakin, "Effect of chemical structure on viscoelasticity and extensional viscosity of drag-reducing cationic surfactant solutions," Langmuir 14, 8-16 (1998).

Mair, R. W., and P. T. Calaghan, "Observation of shear banding in worm-like micelles by NMR velocity imaging," Europhys. Lett. 36, 719-724 (1996).

Mastrangelo, C. H., "Mechanical stability and adhesion of microstructures under capillary forces-Part I: Basic theory," J. Microelectromech. Syst. 2, 33-43 (1993a).

Mastrangelo, C. H., "Mechanical stability and adhesion of microstructures under capillary forces-Part II: Experiments," J. Microelectromech. Syst. 2, 44-55 (1993b).

McKinley, G. H., O. F. Brauner, and M. Yao, "Kinematics of filament stretching in dilute and concentrated polymer solutions," Korea-Australia Rheology Journal 13, 29-35 (2001).

McKinley, G. H., and T. Sridhar, "Filament stretching rheometry,” Annu. Rev. Mater. Sci. 34, 375-415 (2002). 
Odell, J. A., A. Keller, and Y. Rabin, "Flow-induced scission of isolated macromolecules," J. Chem. Phys. 88, 4022-4028 (1988).

Olmsted, P. D., "Dynamics and flow induced phase separation in polymeric fluids," Curr. Opin. Colloid Interface Sci. 4, 95-100 (1999).

Orr, N. V., and T. Sridhar, "Probing the dynamics of polymer solutions in extensional flow using step strain rate experiments," J. Non-Newtonian Fluid Mech. 82, 203-232 (1999).

Perkins, T. T., D. E. Smith, and S. Chu, "Single polymer dynamics in an elongational flow," Science 276, 2016-2021 (1997).

Prudhomme, R. K., and G. G. Warr, "Elongational flow of solutions of rodlike micelles," Langmuir 10, 3419-3426 (1994).

Rehage, H., and H. Hoffmann, "Viscoelastic surfactant solutions: Model systems for rheological research," Mol. Phys. 74, 933-973 (1991).

Rothstein, J. P., and G. H. McKinley, "Extensional flow of a polystyrene Boger fluid through a 4:1:4 axisymmetric contraction/expansion," J. Non-Newtonian Fluid Mech. 86, 61-88 (1999).

Rothstein, J. P., and G. H. McKinley, "The axisymmetric contraction-expansion: The role of extensional rheology on vortex growth dynamics and the enhanced pressure drop," J. Non-Newtonian Fluid Mech. 98, 33-63 (2001).

Rothstein, J. P., and G. H. McKinley, "A comparison of the stress and birefringence growth of dilute, semidilute and concentrated polymer solutions in uniaxial extensional flows," J. Non-Newtonian Fluid Mech. 108, 275-290 (2002a).

Rothstein, J. P., and G. H. McKinley, "Inhomogeneous transient uniaxial extensional rheometry," J. Rheol. 46, 1419-1443 (2002b).

Shikata, T., S. J. Dahman, and D. S. Pearson, "Rheo-optic behavior of wormlike micelles," Langmuir 10, 3470-3476 (1994).

Shikata, T., and T. Kotaka, "Entanglement network of thread-like micelles of a cationic detergent," J. NonCryst. Solids 131-133, 831-835 (1991).

Szabo, P., "Transient filament stretching rheometry I: Force balance analysis," Rheol. Acta 36, 277-284 (1997).

Tripathi, A., G. H. McKinley, M. K. C. Tam, and R. D. Jenkins, "Rheology and dynamics of associative polymers in shear and extension: Theory and experiments," Society of Rheology Annual Meeting, Hilton Head, SC, 11-15, Feb. 2001 (unpublished).

Turner, M. S., and M. E. Cates, "Flow-induced phase transitions in rod-like micelles," J. Phys.: Condens. Matter 4, 3719-3741 (1992).

van Egmond, J. W., "Shear-thickening in suspensions, associating polymers, worm-like micelles and polymer solutions," Curr. Opin. Colloid Interface Sci. 3, 385-390 (1998).

Venerus, D. C., S. H. Zhu, and H.-C. Ottinger, "Stress and birefringence measurements during the uniaxial elongation of polystyrene melts," J. Rheol. 43, 795-813 (1999).

Vinogradov, G. V., A. Y. Malkin, V. V. Volosevitch, V. P. Shatalov, and V. P. Yudin, "Flow, high-elastic (recoverable) deformations and rupture of uncured high molecular weight linear polymers in uniaxial extension,” J. Polym. Sci., Polym. Phys. Ed. 13, 1721-1735 (1975).

Walker, L. M., P. Moldenaers, and J.-F. Berret, "Macroscopic response of wormlike micelles to elongational flow," Langmuir 12, 6309-6314 (1996).

Wedgewood, L. E., D. N. Ostrov, and R. B. Bird, "A finite extensible bead-spring chain model for dilute polymer-solutions,” J. Non-Newtonian Fluid Mech. 40, 119-139 (1991).

Wheeler, E. K., P. Fischer, and G. G. Fuller, "Time-periodic flow induced structures and instabilities in a viscoelastic surfactant solution," J. Non-Newtonian Fluid Mech. 75, 193-208 (1998).

Wheeler, E. K., P. Izo, and G. G. Fuller, "Structure and rheology of wormlike micelles," Rheol. Acta 35, 139-149 (1996).

Yao, M., G. H. McKinley, and B. Debbaut, "Extensional deformation, stress relaxation and necking failure of viscoelastic filaments," J. Non-Newtonian Fluid Mech. 79, 469-501 (1998). 